\title{
Oocyte vitrification: A local validation of the method
}

\author{
Pierre Boyer, Debbie Montjean, Pierre Tourame, Marie-Josée Gervoise-Boyer
}

Service de Médecine et Biologie de la Reproduction, Hôpital Saint Joseph, Marseille, France

Email: pb.boyer@gmail.com

Received 21 February 2013; revised 25 March 2013; accepted 3 April 2013

Copyright (C) 2013 Pierre Boyer et al. This is an open access article distributed under the Creative Commons Attribution License, which permits unrestricted use, distribution, and reproduction in any medium, provided the original work is properly cited.

\begin{abstract}
Is there a really need to validate oocyte vitrification technique in an ART laboratory before establishing it in daily practice? Validation of micromanipulationbased technique, in this case oocyte vitrification, is essential prior to enlarging its use to routine practice. Oocyte vitrification is a new worldwide used technique and legal recently in France. This micromanipulation needs to be performed by a skilled and experienced embryologist and requires an internal assessment in each ART unit before any wide use. We designed a prospective study, from September 2011 to July 2012, using sibling oocytes from women who recovered more than 12 Metaphase II oocytes. A part of freshly recovered oocytes underwent immediate ICSI while the remaining oocytes were vitrified. 87 couples undergoing ICSI were selected based on number of mature oocytes available on the recovery day after denudation. A part of fresh MII oocytes were microinjected and the others were vitrified using an open system (Cryotop $\left.{ }^{\circledR}\right)$. The major criterion of interest was the number of embryo transferred/ number of Metaphase II ratio for after ICSI on fresh oocytes (42/211) versus vitrified/warmed oocytes $(51 / 204)(p>0.05)$. Secondary studied criteria were survival rate $(80.5 \% \pm 26.3 \%)$, fertilization rate $(68.9$ \pm 33.5 ) and finally, cumulative pregnancy rate obtained in this study is $\mathbf{4 0 . 2 \%}$. One of the benefits of such practice is the limitation of embryo freezing. However, the study design delays oocytes warming cycles, due to pregnancies triggered by the transfer of fresh derived oocyte embryos and to the priority to transfer all the frozen embryos before starting oocytes warming. Moreover, no data is available about children' health. Oocyte vitrification represents not only a change in our daily practice to improve cumulative pregnancy rate but also a promising tool to develop egg banking and donation. Clinical Trials Registration number: 209 R02.
\end{abstract}

Keywords: Oocyte; Cryopreservation; Vitrification; ICSI; Sibling Oocyte Study; Method Validation; Pregnancy Rate; Quality

\section{INTRODUCTION}

Oocyte vitrification has been successfully used worldwide in an Assisted Reproductive technology (ART) laboratory for the past years for various purposes ranging from the diminution of frozen embryo number, through egg banking for donation to fertility preservation [1-8]. Interestingly, previous studies have concluded that the results of oocyte vitrification procedure followed by Intra Cytoplasmic Sperm Injection (ICSI) are comparable to fresh microinjection procedure, with regard to fertilization and embryo developmental rates [6]. Furthermore, cumulative ongoing pregnancy rate achieved with oocyte vitrification was shown to be as good as pregnancy rate obtained without the use of oocyte vitrification [8]. These encouraging results show that oocyte vitrification procedure is the cutting-edge technique that will bring about a dramatic change in the field $[9,10]$. French teams were not allowed using oocyte vitrification for years as it was considered as a research on human embryo so far forbidden in France. Therefore, local teams were forced to wait for legal evolution that appeared in a law in July 2011, authorizing oocyte freezing using vitrification in France $[2,11,12]$. Upon the review of the French law, we took on a local evaluation (study No. 2008-A00372-53) to demonstrate that oocyte vitrification can be also widely diffused in our ART laboratory in IVF routine. From September 2011 to July 2012, we proposed couples who required In Vitro Fertilization-Intra Cytoplasmic Sperm Injection (IVF-ICSI) for male infertility reasons to vitrify at least a part of the retrieved oocytes cohort to analyze the efficiency of oocyte vitrification in our laboratory.

\section{METHODS}

\subsection{Patients and Study Design}

Couples included in this study (studied group) had the 
following criteria: Inclusion in an IVF program for medical reasons, male factor infertility requiring the use ICSI, number of metaphase II oocyte recovered was over 12 after eggs decoronisation, no parental constitutional caryotype anomalies, French legal conditions respected for ART treatment especially for viral risk.

The study protocol was proposed to the local ethical committee and accepted under number 208R02. SET (Single Embryo Transfer) was systematically proposed. DET (Duo Embryo transfer) was exceptionally accepted upon clinician's request. Mean age of studied population was $32.2 \pm 4.6$ years. Rank of attempt was $2.1 \pm 1.3$.

\subsection{Ovarian Stimulation, Oocytes and Embryo Following}

Ovarian stimulation was conducted using agonist or antagonist pituitary control. Administration of u-FSH or r-FSH was used to induce ovulation. Oocyte recovery was performed approximately 36 hours after triggering ovulation by hCG administration under transvaginal ultrasound-guided aspiration. For each patient, after oocyte denudation, the cohort of oocytes was divided in two groups. First, at least 6 Metaphase II (MII) oocytes immediately fertilized by ICSI (Fresh oocytes) followed by fresh embryo transfer or, when necessary, conventional embryo slow-freezing. Second, the rest of MII oocytes were vitrified. In the case where no pregnancy was obtained, and all the frozen embryos were transferred, we proposed oocytes warming cycle. The number of oocytes to thaw was decided on the basis of two criteria: fertilization rate of fresh sibling oocytes and oocytes survival rate after warming. Oocytes were warmed 3 by 3 until the desired number of oocytes is obtained for ICSI procedure, in the context of a spontaneous or substituted cycle. Fertilization rate was calculated by number of oocytes showing 2 pronuclei at day $1 \mathrm{vs}$. number of oocytes micro-injected. Embryos were cultured in Global medium microdrops covered with mineral oil at $37.2^{\circ} \mathrm{C}$ under $6 \% \mathrm{CO}_{2}$. Embryo transfer was performed from day 3 to day 5 depending on the embryonic quality, for each fresh transfer. A single senior embryologist assessed embryo quality daily based on morphological and kinetics. Day 5 was privileged due to Single Embryo Transfer preference. Poor quality embryos were discarded. For warming cycles, embryo transfer was also performed at Day 3 or Day 5 according to embryo development. Pregnancies were assessed by blood hCG detection followed by ultrasound assessment of fetal heartbeat.

\subsection{Oocyte Vitrification and Thawing}

Up to 3 oocytes were vitrified on a Cryotop ${ }^{\circledR}$ using Kitazato vitrification kit with the following protocol:
Briefly, Equilibration Solution (ES) and Vitrification Solution (VS) were warmed to room temperature $\left(20^{\circ} \mathrm{C}\right.$ $27^{\circ} \mathrm{C}$ ). The oocytes were subjected to a gradient of ES solution during $15 \mathrm{~min}$, then vitrified in VS solution and immersed into liquid nitrogen within 1 minute.

Oocyte thawing was performed using Kitazato thawing kit following the manufacturer's recommendations: The Thawing Solution (TS) was warmed up to $37^{\circ} \mathrm{C}$ in a dish. The sheet of the Cryotop was quickly immersed into the TS solution. The oocytes were aspirated within the end of the capillary at 1 minute after putting into TS. Oocytes were transferred in Dilution Solution (DS) for 3 minutes and washed twice in two 5-minutes baths in Washing Solutions (WS). Oocytes were incubated at $37^{\circ} \mathrm{C}$ for 2 hours before micro-injection.

\subsection{Statistical Analysis}

Chi-square and Student Test were used and $\mathrm{p}<0.05$ was considered statistically significant.

\section{RESULTS}

\subsection{Organization of the Study}

For the 87 included couples, 1366 oocytes were collected, which represents a mean of 15.7 oocytes per pick up and 1191 MII oocytes, which is an average of 13.7 MII per woman. So far, 648 oocytes have been vitrified and 543 micro-injected immediately after retrieval at Day 0 .

\subsection{Oocyte Vitrification/Thawing Efficiency}

To date, 292 oocytes have been thawed for 33 couples. This allowed us to estimate both the mean survival rate $(80.5 \% \pm 26.3 \%)$ and fertilization rate after oocytes thawing at $68.9 \% \pm 33.5 \%$ compared to $57.7 \% \pm 28.0 \%$ in freshly recovered oocyte sub-group (Figure 1). No statistical difference was found.

Within the sub-group of couples who had at least one warming cycle, the ratio of transferred embryo/MII oocytes was identical in the fresh ICSI cohort oocytes $(42 / 211)$ and in the warmed ICSI cohort oocytes (51/204) ( $p>0.05)$ (Figure 2). This data suggest that oocyte vitrification does not affect embryo development as the same number of embryos is available in both groups for the transfer. A total of 100 fresh oocytes derived embryos and 31 frozen embryos were transferred leading to 22 clinical pregnancies: 17 from fresh oocytes derived embryo transfer and 5 from frozen embryo transfer. Therefore, pregnancy rate is $17 \%$ and $16.1 \%$ for the transfer of fresh oocyte derived embryo and frozen embryo, respectively (Figure 3). 13 clinical pregnancies were registered in the vitrified/warmed oocyte cohort with 53 embryos transferred, leading to a pregnancy rate at $24.5 \%$ per transfer of vitrified/warmed oocyte derived embryos 


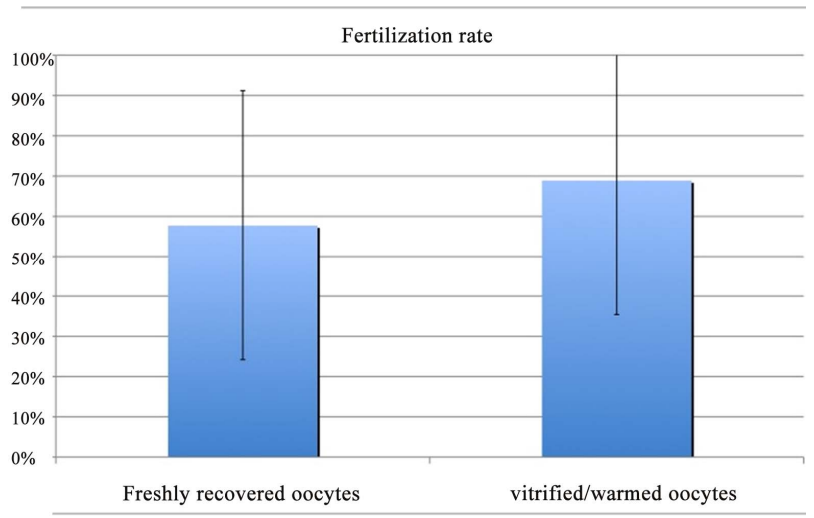

Figure 1. Fertilization rates obtained after microinjection of freshly recovered oocytes vs vitrified/warmed oocytes. No statistical difference was found.

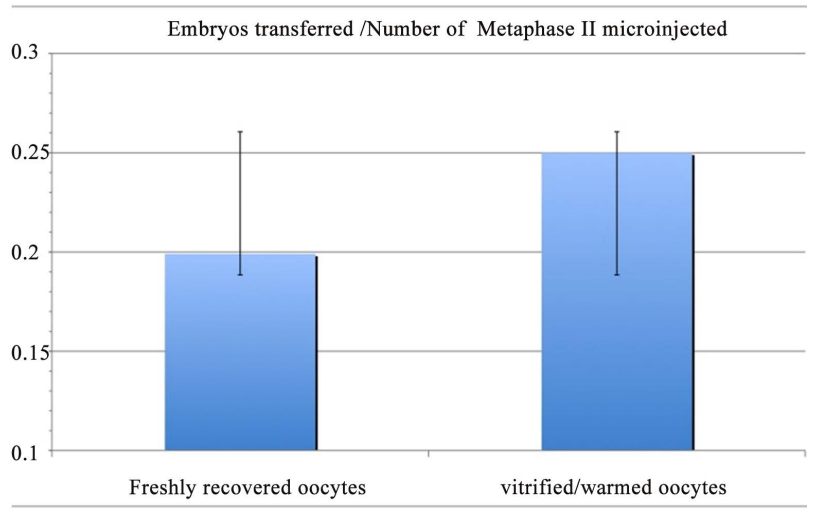

Figure 2. Number of embryos transferred /Number of microinjected metaphase II ratio after microinjection of freshly recovered oocytes vs vitrified/warmed oocytes. No statistical difference was found.

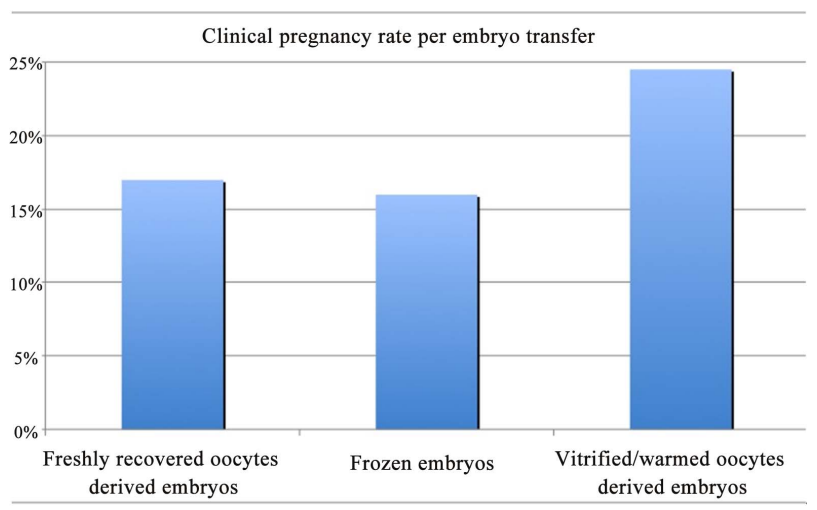

Figure 3. Clinical pregnancy rate per transfer of freshly recovered oocytes derived embryos, frozen embryos and vitrified/warmed oocytes derived embryos. No statistical difference was found.

(there was no thawed embryo transfer after oocyte warming cycles) (Figure 3). Although pregnancy rate obtained after the transfer of embryos deriving from vitrified/warmed oocytes appears to be the highest, no sta- tistical difference was found $(\mathrm{p}>0.05)$. Miscarriage rate is $11.8 \%$ when fresh oocytes derived embryos and frozen embryos are transferred and $7.7 \%$ when vitrified/warmed oocyte derived embryos are transferred. Among the 87 included couples, 62 still have 427 cryopreserved oocytes and 48 cryopreserved embryos remain for 22 couples, suggesting that there are many chances for additional pregnancies. Interestingly, the present evaluation led to a satisfactory cumulative ongoing pregnancy rate: $40.2 \%$.

\section{DISCUSSION}

Several studies were conducted worldwide to test the efficiency of oocyte vitrification as reported in a metaanalysis of randomized controlled trials [2]. As up to July 2011 French authorities did not allow oocyte vitrification, the present study is the first evaluation of this technique in France. Our results show that vitrification prior to oocyte warming and ICSI procedure does not affect fertilization rate, embryo development and pregnancy rate. These data are in line with previous international investigations about oocyte vitrification $[6,8]$.

Historically, oocyte vitrification was applied in oocyte donation program on young women oocytes [13] and in quotidian practice in an Italian IVF centre as an alternative to legal restriction of embryo freezing [6]. The present study promotes a way of performing a local evaluation of such a new approach before using it in routine IVF program in France where embryo freezing is authorized. Exclusively ICSI indication cases were selected because oocyte vitrification technique per se requires decoronised oocytes leading to ICSI for fertilization. This inclusion criterion facilitated the integration of this study in our daily activity with any changes neither in the organization of the laboratory nor in patients' management.

The present study relies on the inclusion of patients who displayed at least 12 oocytes at recovery. However, it is of common knowledge that oocyte quality is negatively correlated with the size of oocyte cohort [14-17]. We chose to split the cohort in the freshly collected part to maintain satisfactory success chances for each couple and to vitrify the rest. The fact that the egg cohort was divided in two groups ensures that the two populations are homogenous and comparable. However, the major limitation is the study relies on the fact that oocytes warming cycles were postponed due to pregnancies obtained after the transfer of fresh derived oocyte embryos and also because of the priority given to the transfer of all the frozen embryos before starting oocytes warming. Therefore, a large amount of oocytes are still cryopreserved.

Like micromanipulations that were developed progressively in the 90s, oocyte vitrification, a complex and 
sophisticated method, should be carefully set up and assessed in each IVF lab despite the actual context of profitability and permanent commercial proposals. The present study was technically conducted by only one senior embryologist to minimize the learning curve impact [18]. This strategy prevents technical drift, thus, efficiency was rapidly reached with satisfactory clinical results. However, hyperstimulation context with the collection of large numbers of oocytes at pick up accounts for our low fertilization rates, equally obtained in both fresh and vitrified/warmed oocytes [14-17].

The relative short lifespan of the mature oocyte is the major parameter to point out because the delay in technique that we apply for embryos is harmful in this case. Therefore, oocyte vitrification was performed concomitantly to ICSI procedure.

Our experience allowed us to identify several advantages for oocyte vitrification technique. Indeed, as oocyte vitrification bypasses embryo freezing, it allows increasing the number of fresh single embryo transfer leading to the same cumulative pregnancy rate as with freshly recovered oocytes. Furthermore, oocyte vitrification practice multiplies the number of ICSI attempts for one oocyte pick up at a limited financial cost.

These encouraging results suggest that oocyte vitrification can be largely used for a panel of purposes including oocyte storing in cases of sperm retrieval failure [19], fertility preservation [20] or egg donation [3,13].

In France, the choosing between open and closed system is still the matter of debate. In the context of fertility preservation we must propose the most effective method to ensure the best success chances. In cancer cases, the major advantage of oocyte vitrification over ovarian tissue preservation is that there is no risk of re-introducing tumoral cells in cancer cases and only the fresh embryo is transferred in the patient's uterus.

Moreover, this technique enlarges the possibilities of fertility preservation to other indications such as severe endometriosis and recurrent cyst that needs a surgical ovarian follicular depletion.

Furthermore, low responders should benefit oocyte vitrification. Indeed, serial oocyte vitrification seems to be a satisfactory approach to improve the management and success rates of such patients [5,21].

Although, further studies are needed to tackle the issue of the health of children born after oocyte vitrification, this new technique appears to be a landmark in IVF laboratories evolution and with the ambition of French authorities to limit the number of cryopreserved embryos [12].

\section{ACKNOWLEDGEMENTS}

We thank Futoshi Inoue and Maki Ogawa from Kitazato BioPharma for providing Cryotop safety Kit. We also thank the clinical staff for ad- dressing the couples to us and allowing them to participate to this program that represents a major evolution for our laboratory.

\section{REFERENCES}

[1] Anderson, R.A. and Wallace, W.H. (2011) Fertility preservation in girls and young women. Clinical Endocrinology, 75, 409-419. doi:10.1111/j.1365-2265.2011.04100.x

[2] Cobo, A. and Diaz, C. (2011) Clinical application of oocyte vitrification: A systematic review and meta-analysis of randomized controlled trials. Fertility and Sterility, 96, 277-285. doi:10.1016/j.fertnstert.2011.06.030

[3] Cobo, A., Remohí, J., Chang, C.C. and Nagy, Z.P. (2011) Oocyte cryopreservation for donor egg banking. Reproductive BioMedicine Online, 23, 341-346. doi:10.1016/j.rbmo.2011.05.014

[4] Cobo, A., de Los Santos, M.J., Castellò, D., Gámiz, P., Campos, P. and Remohí, J. (2012) Outcomes of vitrified early cleavage-stage and blastocyst-stage embryos in a cryopreservation program: Evaluation of 3150 warming cycles. Fertility and Sterility, 98, 1138-1146. doi:10.1016/j.fertnstert.2012.07.1107

[5] Cobo, A., Garrido, N., Crespo, J., José, R. and Pellicer, A. (2012) Accumulation of oocytes: A new strategy for managing low-responder patients. Reproductive BioMedicine Online, 24, 424-432. doi:10.1016/j.rbmo.2011.12.012

[6] Rienzi, L., Romano, S., Albricci, L., Maggiulli, R., Capalbo, A. and Baroni, E., et al. (2010) Embryo development of fresh "versus" vitrified metaphase II oocytes after ICSI: A prospective randomized sibling-oocyte study. Human Reproduction, 25, 66-73. doi:10.1093/humrep/dep346

[7] Rienzi, L., Cobo, A., Paffoni, A., Scarduelli, C., Capalbo, A., Vajta, G., et al. (2012) Consistent and predictable delivery rates after oocyte vitrification: An observational longitudinal cohort multicentric study. Human Reproduction, 27, 1606-1612. doi:10.1093/humrep/des088

[8] Ubaldi, F., Anniballo, R., Romano, S., Baroni, E., Albricci, L., Colamaria, S., Capalbo, A., Sapienza, F., et al. (2010) Cumulative ongoing pregnancy rate achieved with oocyte vitrification and cleavage stage transfer without embryo selection in a standard infertility program. $\mathrm{Hu}$ man Reproduction, 25, 1199-1205. doi:10.1093/humrep/deq046

[9] Boyer, P., Tourame, P. and Gervoise-Boyer, M. (2012) Third millennium assisted reproductive technologies: Time to change our practice. Intech, Rijeka.

[10] Cobo, A. (2012) Oocyte vitrification: A watershed in ART. Fertility and Sterility, 98, 600-601. doi:10.1016/j.fertnstert.2012.07.1096

[11] Kuwayama, M. (2007) Highly efficient vitrification for cryopreservation of human oocytes and embryos: The Cryotop method. Theriogenology, 67, 73-80. doi:10.1016/j.theriogenology.2006.09.014

[12] Boyer, P., Tourame, P. and Le Coz, P. (2010) New assisted reproduction techniques: France, an absent subscriber. Gynécologie Obstétrique \& Fertilité, 38, 561-562. 


\section{doi:10.1016/j.gyobfe.2010.08.035}

[13] Cobo, A., Meseguer, M., Remohí, J. and Pellicer, A. (2010) Use of cryo-banked oocytes in an ovum donation programme: A prospective, randomized, controlled, clinical trial. Human Reproduction, 25, 2239-2246. doi:10.1093/humrep/deq146

[14] Melie, N.A., Adeniyi, O.A., Igbineweka, O.M. and Ajayi, R.A. (2003) Predictive value of the number of oocytes retrieved at ultrasound-directed follicular aspiration with regard to fertilization rates and pregnancy outcome in intracytoplasmic sperm injection treatment cycles. Fertility and Sterility, 80, 1376-1379. doi:10.1016/S0015-0282(03)02213-1

[15] Timeva, T., Milachich, T., Antonova, I., Arabaji, T., Shterev, A. and Omar, H.A. (2006) Correlation between number of retrieved oocytes and pregnancy rate after in vitro fertilization/intracytoplasmic sperm infection. Scientific World Journal, 21, 686-690. doi:10.1100/tsw.2006.145

[16] Van der Gaast, M.H., Eijkemans, M.J., van der Net, J.B., de Boer, E.J., Burger, C.W., van Leeuwen, F.E., et al. (2006) Optimum number of oocytes for a successful first IVF treatment cycle. Reproductive BioMedicine Online, 13, 476-480. doi:10.1016/S1472-6483(10)60633-5

[17] Sunkara, S.K., Rittenberg, V., Raine-Fenning, N., Bhattacharya, S., Zamora, J. and Coomarasamy, A. (2011)
Association between the number of eggs and live birth in IVF treatment: an analysis of 400135 treatment cycles. Human Reproduction, 26, 1768-1774. doi:10.1093/humrep/der106

[18] Dessolle, L., Biau, D.J., de Larouzière, V., Ravel, C., Antoine, J.M., Daraï, E., et al. (2009) Learning curve of vitrification assessed by cumulative summation test for learning curve (LC-CUSUM). Fertility and Sterility, 92, 943-945. doi:10.1016/j.fertnstert.2009.01.133

[19] Song, W.Y., Sun, Y.P., Jin, H.X., Xin, Z.M., Su, Y.C. and Chian, R.C. (2011) Clinical outcome of emergency egg vitrification for women when sperm extraction from the testicular tissues of the male partner is not successful. Systems Biology in Reproductive Medicine, 57, 210-213. doi:10.3109/19396368.2011.566666

[20] Noyes, N., Knopman, J.M., Melzer, K., Fino, M.E., Friedman, B. and Westphal, L.M. (2011) Oocyte cryopreservation as a fertility preservation measure for cancer patients. Reproductive BioMedicine Online, 23, 323-333. doi:10.1016/j.rbmo.2010.11.011

[21] Chung, J.T., Son, W.Y., Zhang, X.Y., Ao, A., Tan, S.L. and Holzer, H. (2012) Normal birth following PGD for reciprocal translocation after serial vitrification of oocytes from a poor responder: A case report. Reproductive BioMedicine Online, 25, 521-526. doi:10.1016/j.rbmo.2012.07.017 